\title{
A Novel Deep Learning based Automatic Auscultatory Method to Measure
}

\section{Blood Pressure}

Fan Pan $^{1}$, Peiyu He* ${ }^{1}$, Fei Chen ${ }^{2}$, Jing Zhang ${ }^{3}, \mathrm{He} \mathrm{Wang}^{4}$ and Dingchang Zheng ${ }^{5}$

${ }^{1}$ College of Electronics and Information Engineering, Sichuan University, Chengdu 610064, China

${ }^{2}$ Southern University of Science and Technology, Shenzhen 518055, China

3 College of Electrical Engineering and Information Technology, Sichuan University, Chengdu 610064, China

${ }^{4}$ School of Computing, University of Leeds, Leeds, LS2 9JT, UK

${ }^{5}$ Faculty of Health, Education, Medicine and Social Care, Anglia Ruskin University, Chelmsford, CM1 1SQ, UK

*Address correspondence to Peiyu He, College of Electronics and Information Engineering, Sichuan University, Chengdu 610064, China. Electronic mail: hpysbsy@163.com 


\section{Abstract}

Background: It is clinically important to develop innovative techniques that can accurately measure blood pressures (BP) automatically.

Objectives: This study aimed to present and evaluate a novel automatic BP measurement method based on deep learning method, and to confirm the effects on measured BPs of the position and contact pressure of stethoscope.

Methods: 30 healthy subjects were recruited. 9 BP measurements (from three different stethoscope contact pressures and three repeats) were performed on each subject. The convolutional neural network $(\mathrm{CNN})$ was designed and trained to identify the Korotkoff sounds at a beat-by-beat level. Next, a mapping algorithm was developed to relate the identified Korotkoff beats to the corresponding cuff pressures for systolic and diastolic BP (SBP and DBP) determinations. Its performance was evaluated by investigating the effects of the position and contact pressure of stethoscope on measured BPs in comparison with reference manual auscultatory method.

Results: The overall measurement errors of the proposed method were $1.4 \pm 2.4 \mathrm{mmHg}$ for SBP and 3.3 $\pm 2.9 \mathrm{mmHg}$ for DBP from all the measurements. In addition, the method demonstrated that there were small SBP differences between the 2 stethoscope positions, respectively at the 3 stethoscope contact pressures, and that DBP from the stethoscope under the cuff was significantly lower than that from outside the cuff by $2.0 \mathrm{mmHg}(\mathrm{P}<0.01)$.

Conclusion: Our findings suggested that the deep learning based method was an effective technique to measure BP, and could be developed further to replace the current oscillometric based automatic blood pressure measurement method.

Keywords: Blood pressure measurement, convolutional neural network, manual auscultatory method, stethoscope position, stethoscope contact pressure. 
Abbreviations list

BP: blood pressure

$\mathrm{CNN}$ : convolutional neural network

SBP: systolic blood pressure

DBP: diastolic blood pressure

KorS: Korotkoff sounds

STFT: Short time Fourier transformation

SD: standard deviation

ANOVA: analysis of variance

AAMI: Association for the Advancement of Medical Instrumentation

BHS: British Hypertension Society

RNN: recurrent neural network

EPSRC: Engineering and Physical Sciences Research Council 


\section{Introduction}

Manual auscultatory and automatic oscillometric techniques are commonly used for non-invasive blood pressure (BP) measurements. Manual auscultatory method is the gold standard for clinical BP measurement,(1) which requires a stethoscope to listen for Korotkoff sounds (KorS) as a cuff encircling the upper arm is deflated. The appearance and disappearance of KorS are associated with systolic and diastolic BPs (SBP and DBP) respectively. Since professional training is required before the operator is competent to perform the manual auscultatory measurement, automatic BP devices have been widely accepted to use at clinical settings or at home because they are easy to use.

The majority of automatic BP devices are based on the oscillometric technique, which estimates SBP and DBP using empirical waveform characteristics ratios derived from the oscillometric waveform envelope.(2) Its main disadvantage is that it does not actually measure BPs, and only estimates BP using an empirical ratio (oscillometric waveform characteristics ratio) calculated from a group of subjects being tested during the device development or validation, leading to inaccurate measurements for individuals. $(3,4)$ The ideal oscillometric waveform characteristics ratios for SBP and DBP are not the same for individuals, and are even different for the same individual under different measurement conditions.(5-8) In addition, movement, muscle contractions, and other artifacts could distort the oscillometric waveform envelope, leading to the inaccurate calculation of the waveform characteristics ratio. $(3,9,10)$ It has also been reported that oscillometric technique is not suitable for patients with cardiac arrhythmia or women with pre-eclampsia.(11,12)

Because of the incapability of highly accurate BP estimation of oscillometric technique, researchers have attempted to employ KorS on automatic BP measurements.(13-17) Determining audible or inaudible stethoscope sound accurately is the key to achieve accurate BP measurement, and most reported methods were based on traditional technology (e.g., 
time-frequency analysis or power spectrum analysis), which are easily influenced by measurement conditions. Hong et al. proposed an advanced signal processing method to convert the main frequencies of KorS into sound pressure levels that were then mapped to equal loudness contours for BP determination. Unfortunately, their method has not been validated on a large set of clinical data.(16) Another challenge for the KorS-based BP measurement methods is the presence of acoustic noise due to microphone sensitivity to patient motion and background noise.(18) Sebald et al. proposed a method to enhance the information signal and cancel the noise signal by filtering the KorS waveforms to create spatially narrowband information signals.(13) Recently, Pan et al. proposed a deep learning based method using convolutional neural network (CNN) to identify KorS from recorded stethoscope sounds during cuff deflation, and assessed the variation of KorS during BP measurement.(19) The effectiveness of using CNN to identify audible or inaudible sound KorS has been assessed. However, the application of CNN to determine BPs has not been fully evaluated.

The main aim of this study was to evaluate a deep learning based automatic BP measurement method with clinical data. Using the reference manual auscultatory method, we have previously investigated the effects of the position and skin contact pressure of stethoscope on measured BPs.(20) In this study, these effects would be quantified using our proposed method and compared with the reference manual auscultatory method to demonstrate the effectiveness of the proposed CNN method.

\section{Methods}

\section{Subjects}


A total of 30 healthy subjects (13 male and 17 female) without any known cardiovascular disease were enrolled in this study, aged from 23 to 63 years. The overall height, weight and arm circumference from all subjects were $169 \pm 8 \mathrm{~cm}, 66 \pm 13 \mathrm{~kg}$ and $27 \pm 3 \mathrm{~cm}$, respectively. All subjects gave their written informed consent to participate in this study. The experiments received ethical permission from the Newcastle \& North Tyneside Research Ethics Committee. The investigation conformed with the principles in the Declaration of Helsinki. All the relevant analysis was performed on anonymised data.

\section{BP Measurement Protocol}

All BP measurements were performed by a trained operator in a quiet and temperature-controlled clinical measurement room. The whole procedure followed the guidelines recommended by the British Hypertension Society (BHS) and American Heart Association.(1,11) Prior to the measurement, each subject was asked to rest on a chair for 5 minutes. During the measurement, they were required not to move and to breathe gently.

As demonstrated in Figure 1, two identical stethoscopes were applied (with one placed under the cuff and the other outside the cuff on the antecubital fossa). During linear cuff deflation, one channel of cuff pressure and two channels of stethoscope sounds from the two stethoscopes were simultaneously and digitally recorded to a data capture computer at a sampling rate of $2000 \mathrm{~Hz}$. 


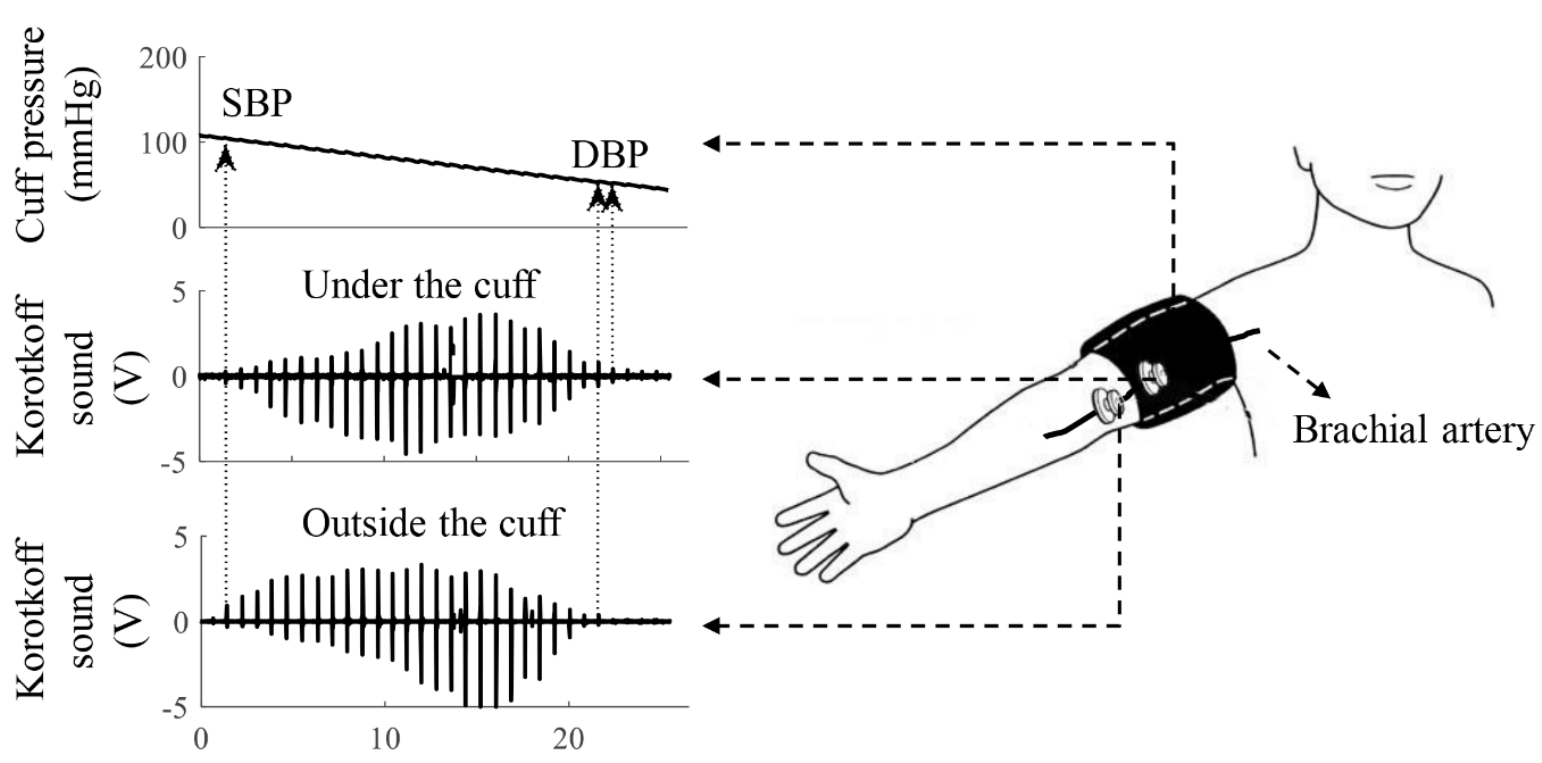

Figure 1. Demonstration of stethoscope sounds recorded from the stethoscope outside (bottom trace) and under (middle trace) the cuff. The corresponding cuff pressure (upper trace) was also recorded simultaneously during cuff deflation.

For each subject, nine BP measurements (from three different stethoscope contact pressures: $0,50,100 \mathrm{mmHg}$, and three repeats) were performed. The three different stethoscope contact pressures were applied sequentially on the stethoscope outside the cuff, with the sequence of these different levels of contact pressure randomized between subjects. A specially designed holder with a spring scale was used to apply different skin contact pressures on the stethoscope head outside the cuff, and the details of the measurement system and protocol were given in our previous publication.(20)

\section{Manual Auscultatory BP Determination}

For each subject, 9 cuff pressure signals and 18 recordings of stethoscope sounds (from 3 repeated recordings of 3 stethoscope contact pressures, and 2 stethoscopes positions) were analyzed off-line. Using a software developed with MATLAB 2011a (MathWork Inc, Massachusetts, USA), all the stethoscope sound recordings were replayed twice on two 
different days to two trained listeners in order to obtain the manual auscultatory BPs. The listener identified the appearance and disappearance of the sounds by clicking the computer mouse. The baseline cuff pressure at which the Korotkoff sound appears was associated with the reference manual SBP, and the cuff pressure at which the Korotkoff sound disappear was associated with the reference manual DBP.

It has been shown in our previous publication that there was no significant BP difference (for both SBP and DBP) between the 2 determinations for each listener and between the 2 listeners (all P > 0.06).(20) Therefore, the average of the four manual BP determinations were calculated and used as the reference BPs for each measurement in this study.

\section{CNN-based BP Determination}

The block diagram in Figure 2 shows the overview of the methodology to determinate BPs from KorS using CNN. The first four steps were to identify the audible KorS, and their details were described in our previous publication.(19) Briefly, the recorded stethoscope signal was firstly segmented into beat-by-beat stethoscope sound frame (1s window with 2,000 sample points per frame) centered with the peak of oscillometric pulse (see Figure 3(a)). During the second step, each frame was converted into matrix 'images' by short time Fourier transformation (STFT) with $60 \mathrm{~ms}$ Hamming window (sampling rate $=2000 \mathrm{~Hz}$ ) and $87 \%$ overlap. Two examples of the converted images are illustrated in Figure 3(b). During the third step, all the frames between the manually determined SBPs and DBPs were labeled as 'Korotkoff' sound beats, while the other frames labeled as 'non-Korotkoff' sound beats. Lastly, a selected set of the labeled frames were trained, and the rest were fed to a CNN classifier to perform automatic identification of Korotkoff and non-Korotkoff beats. A 10-fold cross-validation strategy was used for performance evaluation. Figure 3(c) shows the CNN structure designed in this study, which was convolved by three layers. Each layer consisted of a convolutional layer and a pooling layer in succession. 


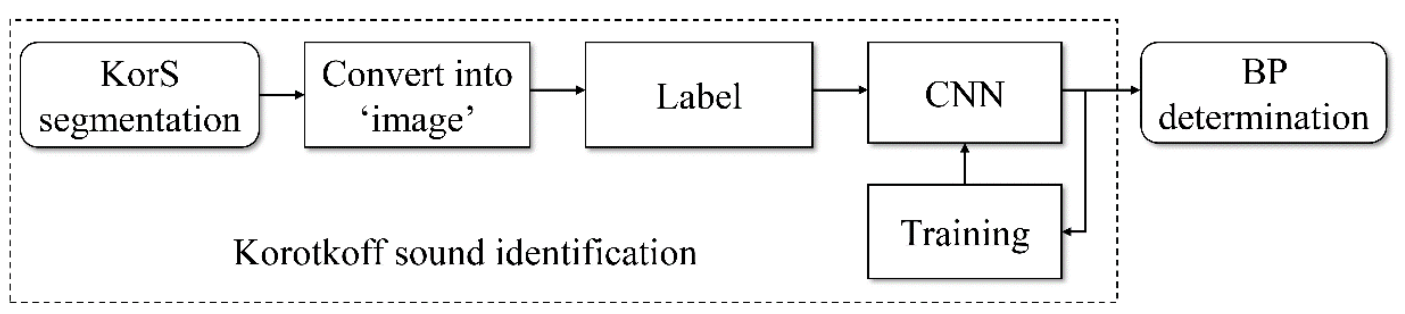

Figure 2. Overview of the methodology to determinate BPs from KorS using CNN.

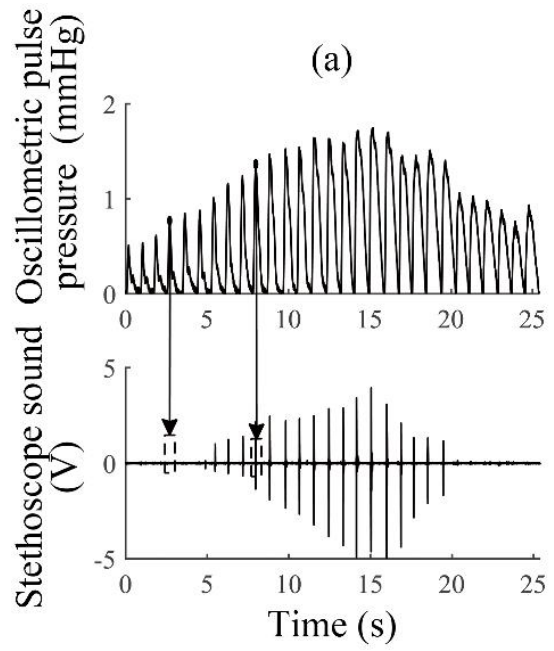

(b)
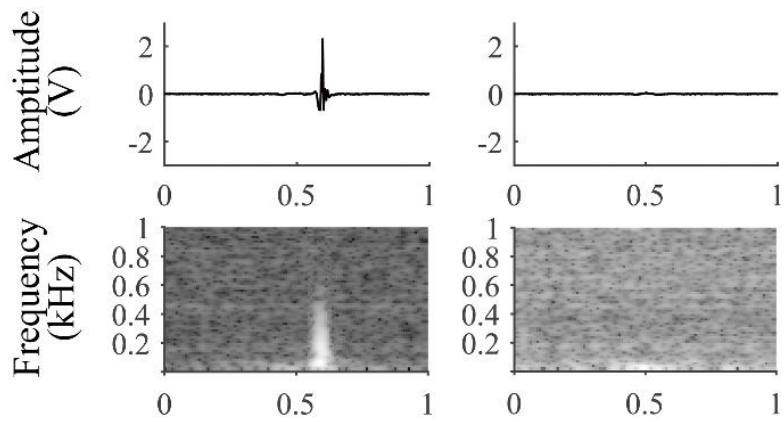

(c)

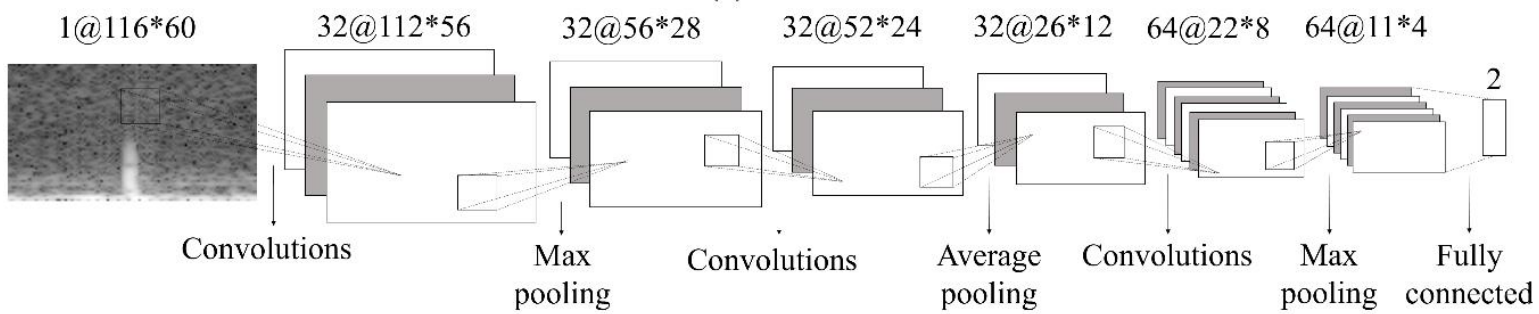

Figure 3. Korotkoff sound identification using CNN. (a) Illustration of stethoscope signal segmentation. Peaks of oscillometric cuff pressure pulses were detected for stethoscope signal segmentation. (b) Converted time-frequency transformation images from Korotkoff (left) and non-Korotkoff (right) sound frames. (c) Illustration of CNN structure with three layers of convolutional and pooling layers.

A mapping algorithm was developed to associate the identified Korotkoff beats for BP determination. Figure 4 illustrates the principle of the mapping algorithm. During the linear cuff pressure deflation of BP measurement, for the ideal situation, it is expected to have continuous non-Korotkoff beats above SBP, followed by continuous Korotkoff beats between 
systole and diastole, and then by continuous non-Korotkoff beats below DBP. In the real scenario, the following BP determination rule was applied to follow the guideline of manual auscultatory BP measurement: SBP was determined with at least two consecutive identified Korotkoff sound beats, and DBP was determined at the point at which all sounds finally disappear completely.(1) After the Korotkoff/non-Korotkoff beats were identified from stethoscope signal for each measurement, the baseline cuff pressures that corresponded to the first and last Korotkoff sound beats were used to determine SBP and DBP. Figure 4(b) gives an example of the application of the BP determination rule.

(a)

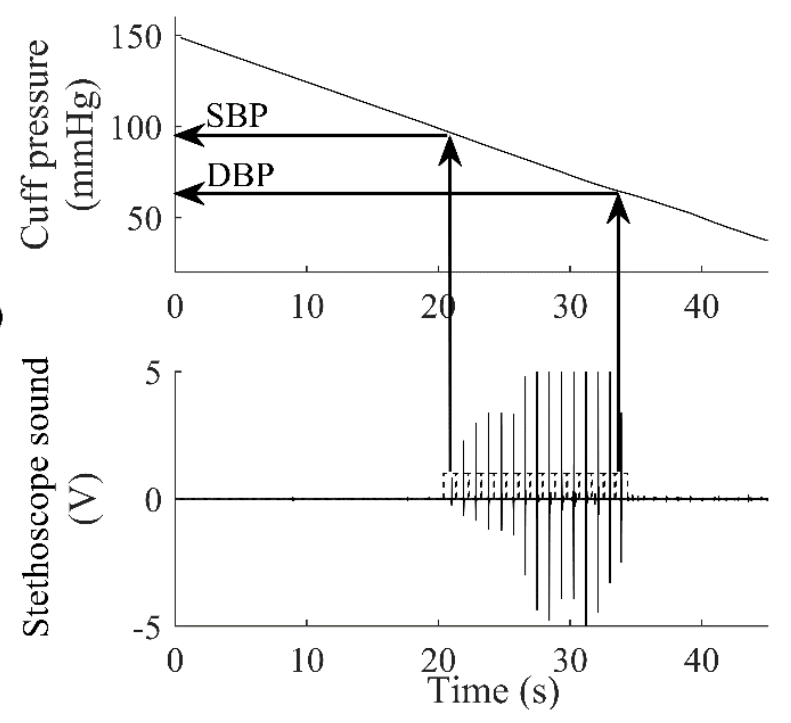

(b)

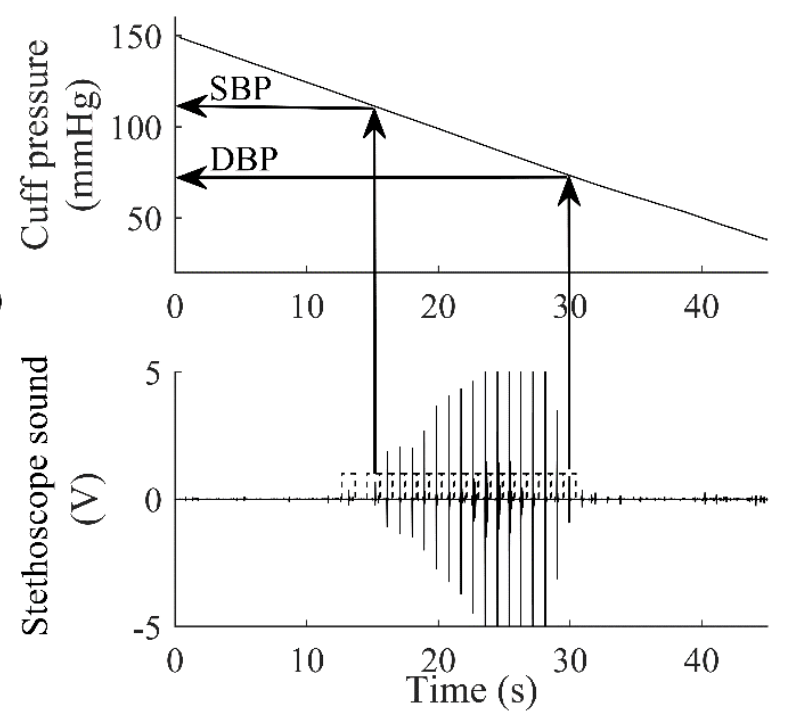


Figure 4. Illustration of the mapping algorithm for BP determination. The beats marked in dashed rectangles are classified as Korotkoff beats. (a) A normal case with continuous Korotkoff beats identified between systole and diastole. (b) A case with one Korotkoff beat following by a non-Korotkoff beat. For this case, the decision rule was applied to determine the SBP from the second Korotkoff beat.

\section{Data and Statistical Analysis}

There were 36 SBP and 36 DBP values from each subject. They were from the measurements with 2 stethoscope positions, 3 contact pressures, 3 repeated recordings and 2 BP determinations by the manual auscultatory method and by the CNN-based method. Their means and standard deviation (SD) were firstly calculated across all subjects. The mean and SD of BP differences between the manual auscultatory method and the CNN-based method were also calculated, with the histograms of BP differences between the two techniques plotted.

Next, Bland-Altman and linear regression analysis were performed to investigate the agreement and relationship between BPs determined by the manual auscultatory method and the CNN-based method. The SPSS Statistics 19 software package (SPSS Inc, Chicago, IL, USA) was employed to perform analysis of variance (ANOVA) analysis to mainly investigate the effects of stethoscope position and contact pressure on the CNN-based BP measurement.

Finally, the BP differences between measurements taken from the stethoscope under and outside the cuff were analyzed and compared between the two methods (the CNN-based method and the manual auscultatory method). All differences were paired values in each subject, and a value of $\mathrm{P}<0.05$ was considered as statistically significant difference.

\section{Results}

\section{Comparison between the CNN-based and Manual Auscultatory Methods}


From all the 540 measurements, the BP differences between the CNN-based method and the manual auscultatory method were $1.4 \pm 2.4 \mathrm{mmHg}$ for SBP and $3.3 \pm 2.9 \mathrm{mmHg}$ for DBP. Figure 5 shows the histogram of the within-subject differences (for both SBP and DBP) between the two methods. Over $99.3 \%$ of SBP difference, and $98.7 \%$ of DBP difference were within $10 \mathrm{mmHg}$. A detailed distribution of these differences is shown in Table 1, which shows the percentage of these differences falling within 5,10 , and $15 \mathrm{mmHg}$.

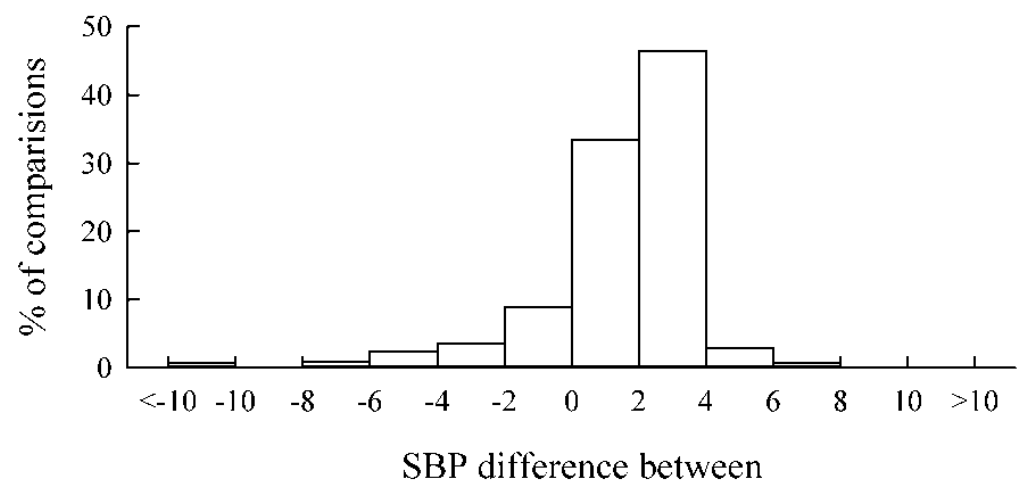

CNN-based method and manual auscultatory method $(\mathrm{mmHg})$

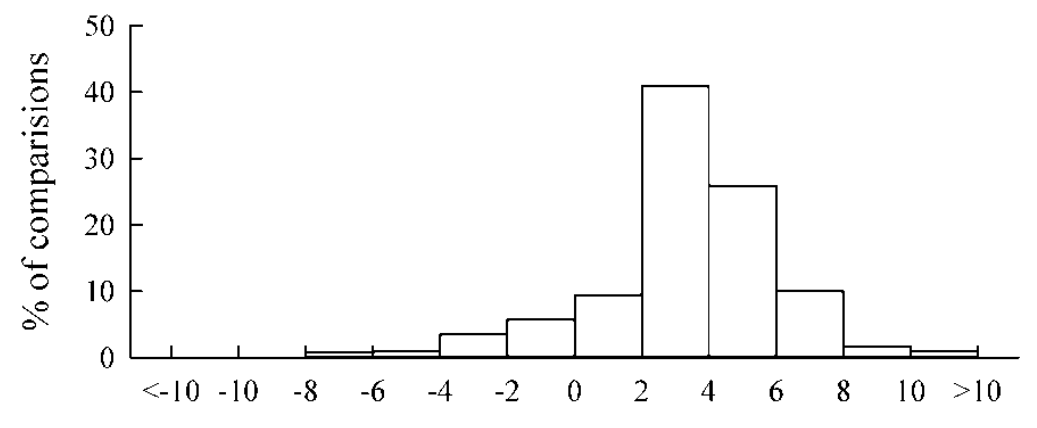

DBP difference between

CNN-based method and manual auscultatory method $(\mathrm{mmHg})$

Figure 5. Histogram of within-subject SBP and DBP differences between the two measurement methods (i.e., the $\mathrm{CNN}$-based method and the manual auscultatory method). A total of 540 comparisons (from 30 subjects, 2 stethoscope positions, 3 contact pressures and 3 repeated recording sessions) were used. 
Table 1. Distribution of BP differences between the CNN-based method and the manual auscultatory method.

\begin{tabular}{cccc}
\hline \hline & Within & Within & Within \\
& $5 \mathrm{mmHg}$ & $10 \mathrm{mmHg}$ & $15 \mathrm{mmHg}$ \\
\hline SBP (\%) & 95.6 & 99.3 & 99.8 \\
DBP (\%) & 76.5 & 98.7 & 100 \\
\hline \hline
\end{tabular}

Figure 6(a) shows the Bland-Altman plots of the SBP and DBP measured by the proposed CNN-based method versus the manual auscultatory method. Their limits of agreement were $4.7 \mathrm{mmHg}$ and $5.7 \mathrm{mmHg}$, respectively for SBP and DBP. The BPs determined by the proposed $\mathrm{CNN}$-based method was in close agreement with those from the manual auscultatory method, as shown in Figure 6(b). The two methods were significantly correlated $(\mathrm{r}=0.98$ for SBP and $\mathrm{r}=0.95$ for DBP) (both $\mathrm{P}<0.001)$.
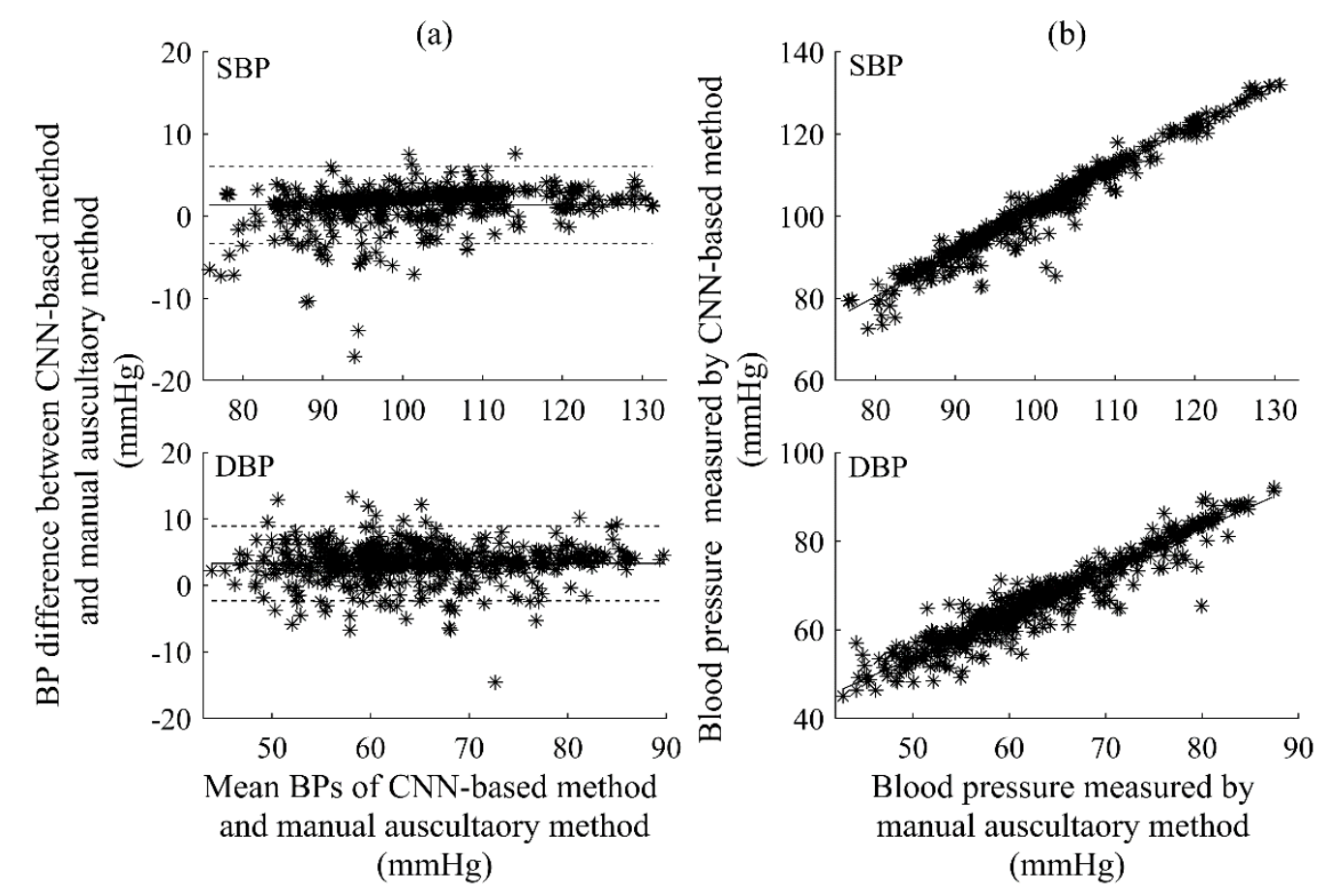

Figure 6. Bland-Altman plots (a) and Regression plots (b) of SBP and DBP from the 
reference the CNN-based method versus manual auscultatory method. The limits of agreement (1.96* SD of BP difference) are given using the dashed lines in the two left sub-figures.

\section{Evaluation of Using the CNN-based Method to Investigate the Effects of Stethoscope Contact Pressure and Position on BP Measurements}

Using the SBP and DBP values estimated by the CNN-based method, variance analysis showed that the effect of stethoscope skin contact pressure on BPs was not statistically significant $(\mathrm{P}=0.22$ for $\mathrm{SBP}$ and $\mathrm{P}=0.72$ for $\mathrm{DBP})$. In terms of the effect of stethoscope position on the CNN-based BP measurements, there were small BP differences between the two stethoscope positions. As shown in Figure 7, 96\% of SBP measurements and $61 \%$ of DBP measurements had a difference of no more than $3 \mathrm{mmHg}$. More specifically, as shown in Figure 8, at each of three levels of stethoscope contact pressure (i.e., 0, 50 and $100 \mathrm{mmHg}$ ), SBPs taken from the stethoscope under the cuff were $0.4,0.6$ and $0.8 \mathrm{mmHg}$ higher than those from outside the cuff. Their corresponding DBPs from the stethoscope under the cuff were statistically significantly lower by $-2.9,-3.0$ and $-2.0 \mathrm{mmHg}$ than those from outside the cuff (all P $<0.01)$. 

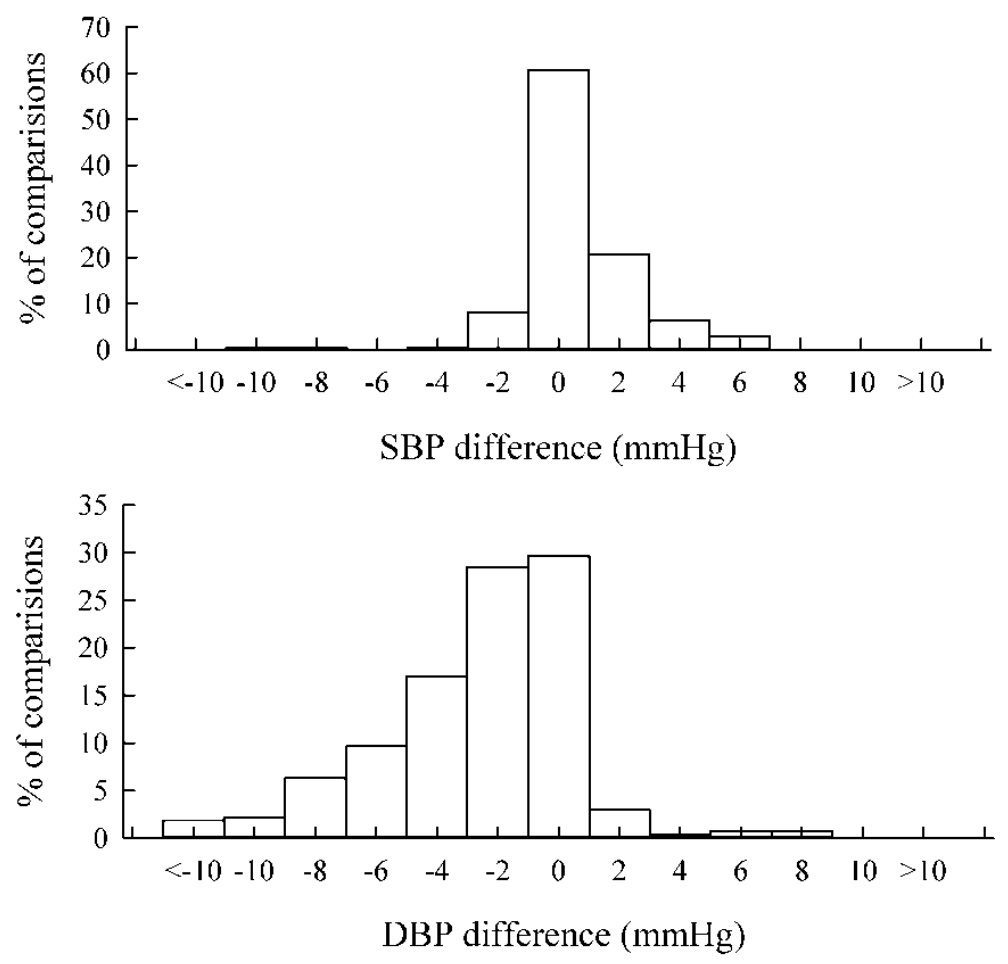

Figure 7. Histogram of within-subject SBP and DBP differences between the measurements taken under the cuff and outside the cuff by the CNN method. A total of 540 comparisons (from 30 subjects, 3 contact pressures, 2 position, and 3 repeats) were used.

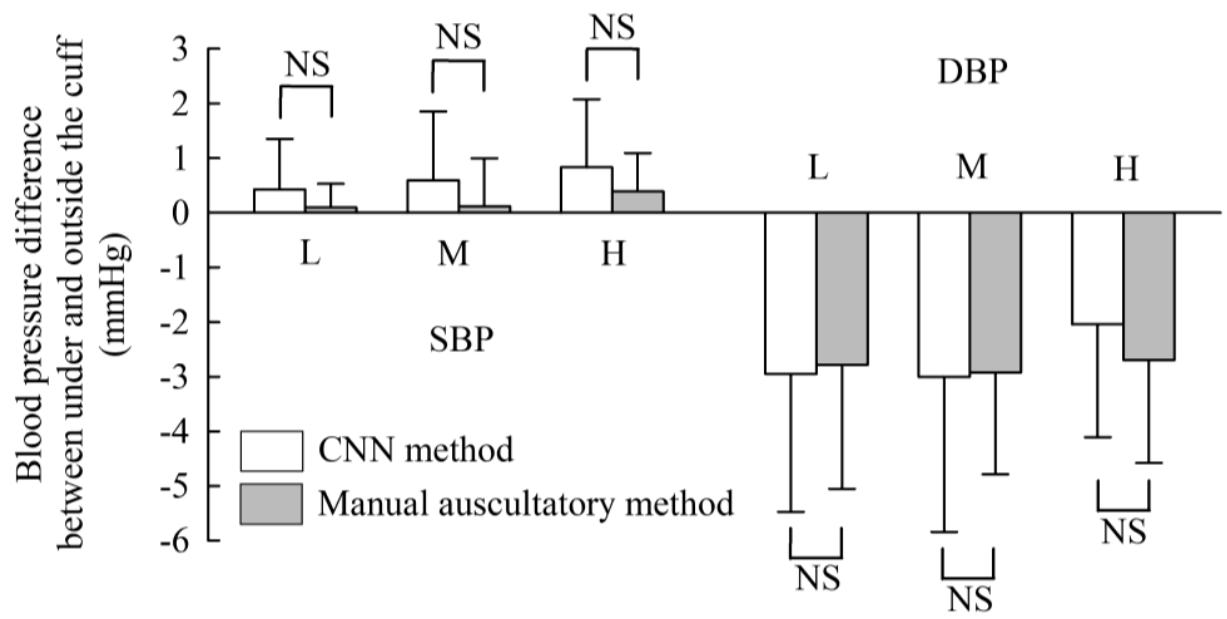

Figure 8. Overall mean difference and SD of within-subject SBP and DBP differences between the measurements taken from the stethoscopes under and outside the cuff. The BP values determined by both the CNN method and the manual auscultatory method are given, separately for the three different contact pressures on the outside stethoscope head (L: 
0mmHg, M: $50 \mathrm{mmHg}$, and $\mathrm{H}: 100 \mathrm{mmHg}$ ). 'NS': Not significant between the paired comparisons, $\mathrm{P} \geq 0.05$.

More importantly, as demonstrated in Figure 8, the effects of stethoscope position on manual BPs and on CNN-based BP determination were not significantly different (all P > 0.05). This applied to all the three stethoscope contact pressures (all $\mathrm{P}>0.05$ ), indicating that the CNN-based method was an effective technique to differentiate the effects of position and contact pressure of stethoscope on clinical BP measurements.

\section{Discussion}

This study evaluated the performance of a CNN-based method for BP measurement in comparison with the reference manual auscultatory method. To the best of our knowledge, this is the first study that the CNN method was developed for automatic auscultatory BP measurement.

The CNN-based method achieved BP measurement errors of $1.4 \pm 2.4 \mathrm{mmHg}$ for SBP and $3.3 \pm 2.9 \mathrm{mmHg}$ for DBP. This level of accuracy was within the requirement of BP device validation from the Association for the Advancement of Medical Instrumentation (AAMI) (average difference no greater than $5 \mathrm{mmHg}$ and $\mathrm{SD}$ no greater than $8 \mathrm{mmHg}$ ). It is also within the Grade A standard for BP device by BHS (see Figure 5 and Table 1). However, it was observed that both SBP and DBP determined by the CNN-based method were significant higher than the manual auscultatory method. This is because the manual auscultatory BPs in this study were obtained by clicking the computer mouse after determining the appearance and disappearance of the KorS, which would cause some delays in BP determinations, consequently generating systematic difference in the data analysis. 
It was also observed that $0.7 \%$ of SBP differences and $2.6 \%$ of DBP difference were over $8 \mathrm{mmHg}$ between the two methods. Figure 9 demonstrates an example that the SBP difference is over $17 \mathrm{mmHg}$. It can be seen that, due to the background noise, the third KorS beat has not been successfully detected by the CNN-based method, indicating that noise is an important factor influencing the accuracy of KorS identification. In future studies, additional pre-processing algorithms may be required to achieve better KorS identification.

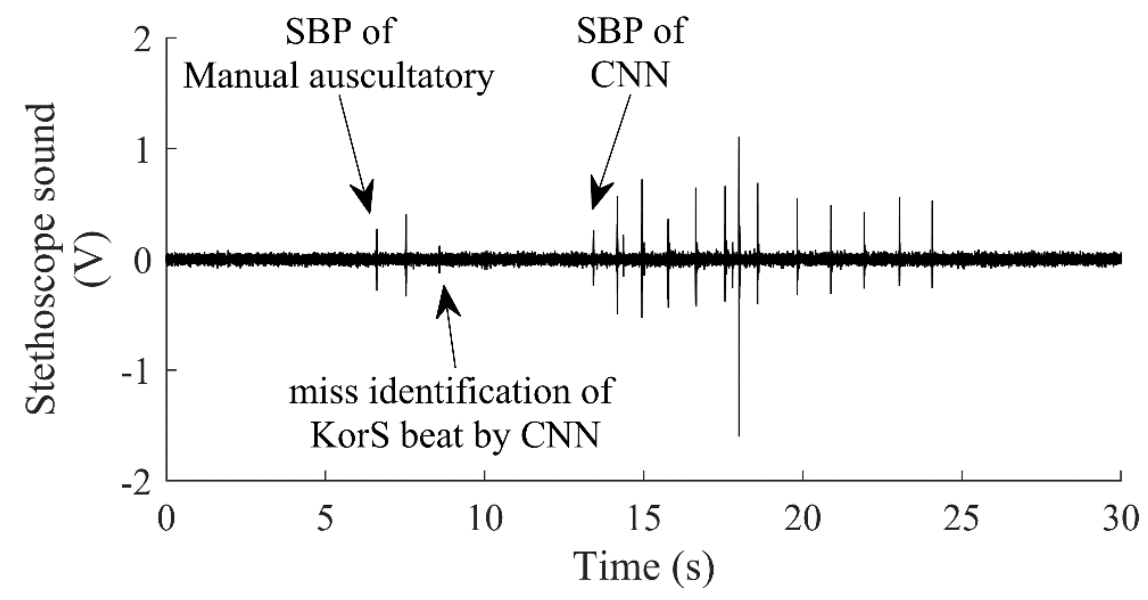

Figure 9. An example of an auscultatory gap, which illustrates the reason for the large SBP difference between the CNN-based method and the manual auscultatory method.

More interestingly, the effects of stethoscope position and skin contact pressure on measured BPs have been confirmed by applying the CNN-based method. There was no significant difference on the effects determined by the manual auscultatory method and by the CNN-based method, indicating that the proposed CNN-based BP method had a good agreement with the manual auscultatory method to differentiate the effects of position and contact pressure of stethoscope on clinical BP measurements.(20) It is therefore concluded that the CNN-based method is an effective technique for non-invasive BP measurement.

It is generally accepted that the phase V of KorS (KorS disappear completely) should be used to determine DBP. In situations where KorS are audible even after the complete cuff deflation (for example, in pregnant women, patients with arteriovenous fistulas, and aortic 
insufficiency), the phase IV should be used.(12,21,22) It would be therefore clinically useful to identify the different phases automatically. Our proposed CNN-based method has not been evaluated to differentiate the different phases of KorS because of the limited number of frames for each phase. A future study with a large data set for training can be designed to evaluate the effectiveness of using $\mathrm{CNN}$ for differentiating the different phases of KorS.

One limitation of this study is that as the manual auscultatory BPs determined by electronic playback may not correspond exactly to clinical auscultation. However, in this study, during the auscultatory BPs determination by playback, all the sound recordings were replayed twice (on 2 different days) to 2 trained listeners to avoid the potential BP measurement bias. Secondly, a decision rule was applied individually to determine which identified Korotkoff/non-Korotkoff beats should be included for BP determination, which is inconvenient. In the future, the recurrent neural network $(\mathrm{RNN})(23)$ could be introduced to include the prior knowledge (such as the five distinct Korotkoff phases as mentioned above) and context (consists of continuous non-KorS beats, followed by continuous KorS beats, and then by continuous non-KorS beats) to achieve automatic and direct BP determination from neural network. Furthermore, a proper clinical study with AAMI/BHS/ISO validation protocol is also required.(24) Thirdly, noise and KorS waveform characteristics (intensity, morphology and spectral properties) may affect the detection of Korotkoff sounds. (25) It is worth investigating the effect of these factors on the outcome of CNN-based BP measurement method in future studies.

In summary, this study provided scientific evidence to demonstrate that the proposed CNN-based BP measurement method was an effective technique to determine BPs, as evidenced by the achievement of high measurement accuracy and agreement with the reference manual auscultatory method and by differentiating the effects of position and contact pressure of stethoscope on clinical BP measurements. 


\section{Authors' contributions}

All authors have made a substantial, direct, intellectual contribution to this study. Fan: study concept and design, algorithm design, data analysis and drafting of the manuscript. Peiyu and Dingchang: analysis and interpretation of data, critical revision of the manuscript for important intellectual content. Fei, Jing and He: critical revision of the manuscript for important intellectual content.

\section{Acknowledgements}

The experiment was conducted with the support from the Engineering and Physical Sciences Research Council (EPSRC) Healthcare Partnership Award (reference number EP/I027270/1). Fan Pan was sponsored by a visiting scholarship to study at Newcastle University between 2012 and 2013.

\section{Statement on conflicts of interest}

All authors declare that we have no conflicts of interest, and this study has no relationships with industry. 


\section{References}

1. Beevers G, Lip GY, O'Brien E. ABC of hypertension: Blood pressure measurement. Part II-conventional sphygmomanometry: technique of auscultatory blood pressure measurement. BMJ 2001;322:1043-7.

2. Baker PD, Westenskow DR, Kuck K. Theoretical analysis of non-invasive oscillometric maximum amplitude algorithm for estimating mean blood pressure. Med Biol Eng Comput 1997;35:271-8.

3. van Montfrans GA. Oscillometric blood pressure measurement: progress and problems. Blood Press Monit 2001;6:287-90.

4. Alpert BS, Quinn D, Gallick D. Oscillometric blood pressure: a review for clinicians. J Am Soc Hypertens 2014;8:930-8.

5. Anastas ZM, Jimerson E, Garolis S. Comparison of noninvasive blood pressure measurements in patients with atrial fibrillation. J Cardiovasc Nurs 2008;23:519-24; quiz 525-6.

6. Lodi CA, Estridge C, Ghidini C. In vitro and in vivo evaluation of an oscillometric device for monitoring blood pressure in dialysis patients. Nephrol Dial Transplant 2007;22:2950-61.

7. Herakova N, Nwobodo NHN, Wang Y, Chen F, Zheng D. Effect of respiratory pattern on automated clinical blood pressure measurement: an observational study with normotensive subjects. Clin Hypertens 2017;23:15.

8. Zheng D, Giovannini R, Murray A. Effect of respiration, talking and small body movements on blood pressure measurement. J Hum Hypertens 2012;26:458-62.

9. Amoore JN, Lemesre Y, Murray IC et al. Automatic blood pressure measurement: the oscillometric waveform shape is a potential contributor to differences between oscillometric and auscultatory pressure measurements. J Hypertens 2008;26:35-43.

10. Lin CT, Liu SH, Wang JJ, Wen ZC. Reduction of interference in oscillometric arterial blood pressure measurement using fuzzy logic. IEEE Trans Biomed Eng 2003;50:432-41.

11. Pickering TG, Hall JE, Appel LJ et al. Recommendations for blood pressure measurement in humans and experimental animals: part 1: blood pressure measurement in humans: a statement for professionals from the Subcommittee of Professional and Public Education of the American Heart Association Council on High Blood Pressure Research. Circulation 2005;111:697-716.

12. O'Brien E, Asmar R, Beilin L et al. European Society of Hypertension recommendations for conventional, ambulatory and home blood pressure measurement. J Hypertens 2003;21:821-48.

13. Sebald DJ, Bahr DE, Kahn AR. Narrowband auscultatory blood pressure measurement. IEEE Trans Biomed Eng 2002;49:1038-44.

14. Park DK, Oh HS, Kang JH, Kim IY, Chee YJ, Lee JS. Novel method of automatic auscultation for blood pressure measurement using pulses in cuff pressure and korotkoff sound. 2008 Computers in Cardiology, 2008:181-184.

15. Xiong L, Panicker GV, Im JJ. A study for the development of K-sound based automatic blood pressure device using PVDF film. Conf Proc IEEE Eng Med Biol Soc 2016;2016:255-258.

16. Hong SJ, Lee JS, Oh JH, Chee YJ, Jang DP, Kim IY. Automatic assessment of blood pressure for Korotkoff sounds on the basis of human hearing threshold. Blood Press Monit 2017;22:364-370.

17. Regueiro-Gomez A, Pallas-Areny R. A new method for automated blood pressure measurement. Physiol Meas 1998;19:205-12.

18. Nitzan M. Automatic noninvasive measurement of arterial blood pressure. IEEE Instrumentation \& Measurement Magazine 2011;14:32-37.

19. Pan F, He P, Liu C, Li T, Murray A, Zheng D. Variation of the Korotkoff Stethoscope Sounds During Blood Pressure Measurement: Analysis Using a Convolutional Neural Network. IEEE J Biomed Health Inform 2017;21:1593-1598.

20. Pan F, Zheng DC, He PY, Murray A. Does the Position or Contact Pressure of the Stethoscope Make Any Difference to Clinical Blood Pressure Measurements An Observational Study. Medicine 2014;93.

21. Perloff D, Grim C, Flack J et al. Human blood pressure determination by sphygmomanometry. Circulation 1993;88:2460-70.

22. Shennan A, Gupta M, Halligan A, Taylor DJ, de Swiet M. Lack of reproducibility in pregnancy of Korotkoff phase IV as measured by mercury sphygmomanometry. Lancet 1996;347:139-42.

23. Gelly G, Gauvain J. Optimization of RNN-Based Speech Activity Detection. IEEE/ACM Transactions on 
Audio, Speech, and Language Processing 2018;26:646-656.

24. Stergiou GS, Alpert BS, Mieke S, Wang J, O'Brien E. Validation protocols for blood pressure measuring devices in the 21st century. J Clin Hypertens (Greenwich) 2018;20:1096-1099.

25. Celler BG, Le P, Basilakis J, Ambikairajah E. Improving the quality and accuracy of non-invasive blood pressure measurement by visual inspection and automated signal processing of the Korotkoff sounds. Physiological measurement 2017;38:1006-1022. 


\section{Summary points}

\section{What is already known:}

- Deep learning technologies have achieved very high accuracy at a broad variety of medical tasks

- Blood pressure measurement is often influenced by the measurement condition

\section{What this study has added:}

- Proposed a deep learning based automatic auscultatory method to measure blood pressure

- Comprehensive evaluation of the performance of the deep learning based blood pressure measurement method 